Article

\title{
The Vertical Dynamic Properties of Flexible Footbridges under Bipedal Crowd Induced Excitation
}

\author{
Yan-an Gao ${ }^{1,2, *}$, Juan Wang ${ }^{2}$ and Min Liu ${ }^{3}$ \\ 1 Faculty of Architecture and Civil Engineering, Huaiyin Institute of Technology, Huaian 223001, China \\ 2 School of Civil Engineering, Beijing Jiaotong University, Beijing 100044, China; juanwang@bjtu.edu.cn \\ 3 School of Civil Engineering, Chongqing University, Chongqing 400044, China; min_liu@bjtu.edu.cn \\ * Correspondence: gaoyn_edu@sina.com; Tel.: +86-13240726429 or +86-15161750325
}

Academic Editor: César M. A. Vasques

Received: 27 March 2017; Accepted: 16 June 2017; Published: 1 July 2017

\begin{abstract}
The excessive vibration of footbridges caused by walking pedestrians has generated great public concern in recent years. However, it has not been explored how crowd size quantitatively influences structural dynamic properties, including human dynamic properties. This paper provides an alternative method for understanding how crowds excite the excessive sway motion of a large-span structure when walking on it. In this study, pedestrians are modeled by a walking dynamic bipedal system. The crowd-structure system is established based on the bipedal model, for which the dynamic properties from pedestrians are considered. The vertical dynamic property equations of structure are calculated under crowd-induced vibration, based on the assumption of a uniformly distributed crowd. Through the proposed framework, the changes of frequency and damping of structure induced by walking pedestrians are studied. The increase of the crowd size can reduce structural frequency, but increase its damping. The impact tendency of crowd size on structural dynamic properties is consistent with measured results. This research provides insight as to how crowd size quantitatively influences the change of structural dynamic properties.
\end{abstract}

Keywords: bipedal crowd model; vertical vibration; dynamic properties

\section{Introduction}

Long-span structures, e.g., footbridges, have become prevalent all over the world, and the excessive dynamic vibrations induced by locomotive crowds are a crucial maintenance concern for their life span. Full-scale dynamic measurements on service structures has shown that humans can change structural vertical dynamic properties [1,2], which has also been confirmed by a measurement experiment on a beam bearing different numbers of persons in Shahabpoor et al. [3]. To explain the vibration mechanism induced by humans, some human-structure models have been proposed, including a one-person model [4,5] and a crowd model [6-9]. Zhou et al. [6,7] studied vertical dynamic characteristics of structure under a modeled human oscillator. Caprani and Ahmadi [8] proposed a moving oscillator to simulate walking pedestrians and further studied the influence of structure frequency and damping variations. It is noted that crowd size may significantly affect variation of the dynamic properties of footbridges. Some crowd-structure interaction models have also been proposed to explore this mechanism. Nimmen et al. [9] proposed two reduced-order models to approximate the dynamic behavior of a full-order coupled crowd-structure interaction system. Alonso et al. [10] also recommended a vertical crowd-structure interaction model to analyze the effect of modal properties variations of a footbridge. To consider gait details, a human-structure interaction with bipedal pedestrian model was proposed by Qin et al. [4,5]. Gao and Yang [11-13] extended this model to a crowd-structure interaction, based on a stability improvement technique. Although the bipedal pedestrian model is successfully applied for crowd-structure interaction in Gao 
and Yang [11-13], the theoretical mechanisms between crowd size and structural modal properties by using this model have not yet been reported. Shahabpoor et al. [3] studied the effect of crowd size on vertical modal parameters of an occupied structure by extensive Frequency Response Function (FRF)-based modal identification tests. Although they quantitatively researched the impact of the number of occupants on structural dynamic properties, one applicable formula which includes human dynamic properties for vibrational control between crowd scale and structural modal characteristics, is absent. Considering that the dynamic properties of humans are crucial to elucidating the variation of structural modal properties, it is therefore imperative to provide a quantitative estimation between crowd size and modal properties of structure.

In this paper, the theoretical formulae between crowd size and vertical modal properties are proposed as an extension of the study in Gao and Yang [11-13]. Firstly, the dynamic equilibrium equation of a structure is established by considering the vertical interaction between bipedal crowd and structure. Secondly, an assumed uniform distribution of a walking crowd is used to calculate the crowd size by Taylor Expansion. Thirdly, the theoretical modal property formulas are derived, based on the assumption of a synchronous movement behavior. Finally, a numerical example is used to analyze the effect of crowd size on structural modal properties.

\section{Dynamic Excitation Mechanism}

The bipedal pedestrian structure system [13] is shown in Figure 1. The left end of the beam is defined as the origin of a planar coordinate system $x-0-z ; L_{B}$ is span length; $m^{(q)}$ is the lump mass of the bipedal model; the superscript " $q$ " means the ordinal number of pedestrians; $x^{(q)}$ and $z^{(q)}$ are longitudinal and vertical displacements of the center of mass $(\mathrm{CoM})$, respectively; $x_{t}^{(q)}$ and $x_{l}^{(q)}$ are the trialing and leading footholds positions, respectively; $\left(x_{l}^{(q)}, t\right)$ and $\left(x_{t}^{(q)}, t\right)$ are structural vertical displacements in the leading and trialing footholds, respectively.

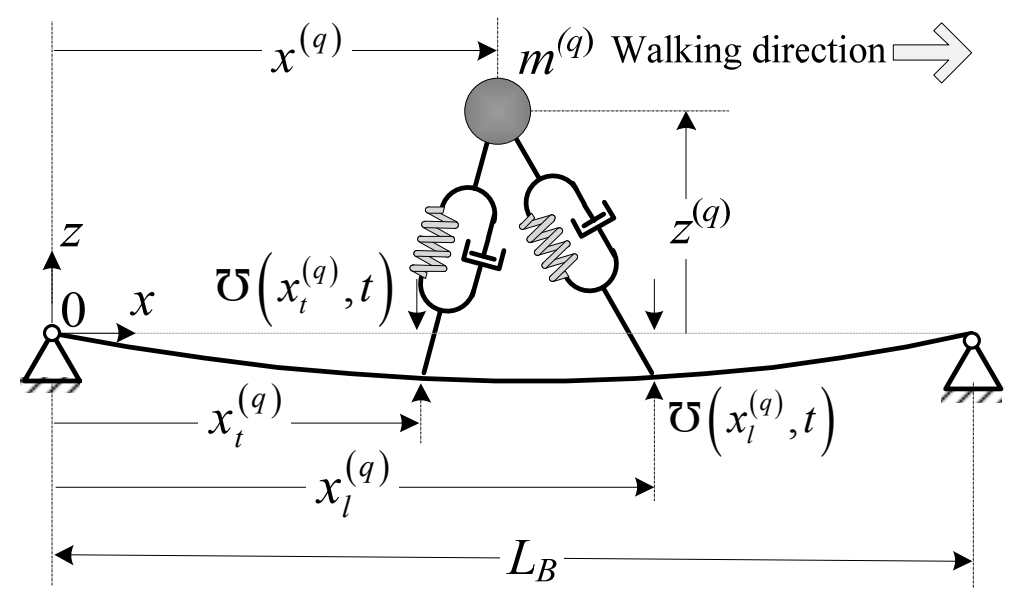

Figure 1. Crowd-structure interaction model.

By only considering the first modal property, the dynamic Equation (8a) from the study [13] can be rewritten as:

$$
\begin{aligned}
& M_{1} \ddot{Y}_{1}(t)+2 \xi_{1} \omega_{1} M_{1} \dot{Y}_{1}(t)+\omega_{1}^{2} M_{1} Y_{1}+\sum_{q=1}^{\chi}\left(c_{z z}^{(q)} \otimes \Phi_{1,1}\right) \dot{Y}_{1}(t) \\
& -\sum_{q=1}^{\chi}\left(c_{z z}^{(q)} \otimes \phi_{1}\right) \dot{z}^{(q)}-\sum_{q=1}^{\chi}\left(k_{z}^{(q)} \otimes \phi_{1}\right) z^{(q)}=\sum_{q=1}^{\chi}\left(c_{x z}^{(q)} \otimes \phi_{1}\right) \dot{x}^{(q)}
\end{aligned}
$$

where $M_{1}$ is modal mass of structure; $\xi_{1}$ and $\omega_{1}$ are the damping ratio and circular frequency of structure, respectively; $Y_{1}, \dot{Y}_{1}$ and $\ddot{Y}_{1}$ are general displacement, velocity and acceleration, respectively; 
$\phi_{1}(x)=\sin \left(\pi x / L_{B}\right)$ and $\Phi_{1,1}(x)=\phi_{1}^{2}(x)$ are the structural modal [13]. The tensor symbols $c_{z z}^{(q)} \otimes \Phi_{1,1}$ and $c_{x z}^{(q)} \otimes \phi_{1}$ are shown as

$$
\begin{gathered}
c_{z z}^{(q)} \otimes \Phi_{1,1}=c_{l z z}^{(q)} \Phi_{1,1}\left(x_{l}^{(q)}\right)+c_{t z z}^{(q)} \Phi_{1,1}\left(x_{t}^{(q)}\right) \\
c_{x z}^{(q)} \otimes \phi_{1}=c_{l x z}^{(q)} \phi_{1}\left(x_{l}^{(q)}\right)+c_{t x z}^{(q)} \phi_{1}\left(x_{t}^{(q)}\right)
\end{gathered}
$$

The leading and trailing leg damping coefficients are shown as

$$
\begin{aligned}
& c_{l z z}^{(q)}=c_{l}^{(q)}\left(L_{l z}^{(q)} / L_{l}^{(q)}\right)^{2}, c_{l x z}^{(q)}=c_{l}^{(q)}\left(L_{l x}^{(q)} L_{l z}^{(q)}\right) /\left(L_{l}^{(q)}\right)^{2} \\
& c_{t z z}^{(q)}=c_{t}^{(q)}\left(L_{t z}^{(q)} / L_{t}^{(q)}\right)^{2}, c_{t x z}^{(q)}=c_{t}^{(q)}\left(L_{t x}^{(q)} L_{t z}^{(q)}\right) /\left(L_{t}^{(q)}\right)^{2}
\end{aligned}
$$

where $c_{l}^{(q)}$ and $c_{t}^{(q)}$ are the leading and trialing leg damping, respectively; $L_{l}^{(q)}$ and $L_{t}^{(q)}$ are the leading and trialing leg lengths, respectively. The expressions of $L_{l x}^{(q)}, L_{l z}^{(q)}, L_{t x}^{(q)}$ and $L_{t z}^{(q)}$ can be found in the study [13]. The tensor product $k_{z}^{(q)} \otimes \phi_{1}$ is shown as:

$$
k_{z}^{(q)} \otimes \phi_{1}=k_{l z}^{(q)} \phi_{1}\left(x_{l}^{(q)}\right)+k_{t z}^{(q)} \phi_{1}\left(x_{t}^{(q)}\right)
$$

where $k_{l z}^{(q)}$ and $k_{t z}^{(q)}$ are given by

$$
k_{l z}^{(q)}=k_{l}^{(q)}\left(1-L_{0}^{(q)} / L_{l}^{(q)}\right)\left(1-{ }_{l}^{(q)} / z^{(q)}\right), k_{t z}^{(q)}=k_{t}^{(q)}\left(1-L_{0}^{(q)} / L_{t}^{(q)}\right)\left(1-{ }_{t}^{(q)} / z^{(q)}\right)
$$

$k_{l}^{(q)}$ and $k_{t}^{(q)}$ are the stiffness of leading and trialing legs, respectively. $L_{0}^{(q)}$ is the relaxed length of the leg. Substituting Equations (2)-(7) into Equation (1), the dynamic equation of a crowd-structure system can be expressed as:

$$
\ddot{Y}_{1}(t)+2 \widetilde{\xi} \widetilde{\omega} \dot{Y}_{1}(t)+\widetilde{\omega}^{2} Y_{1}=\widetilde{F}
$$

where $\widetilde{\xi}$ and $\widetilde{\omega}=2 \pi \widetilde{f}$ are the damping ratio and circular frequency of the structure induced by humans; $\widetilde{f}$ is the structure frequency induced by humans; $\widetilde{F}$ is the excited force. These are obtained by:

$$
\begin{gathered}
\widetilde{\xi}=\xi_{1} \frac{f_{1}}{\widetilde{f}}+\frac{1}{4 \pi M_{1} \widetilde{f}} \sum_{q=1}^{\chi}\left(c_{z z}^{(q)} \otimes \Phi_{1,1}\right) \\
\widetilde{f}=\sqrt{f_{1}^{2}-\frac{1}{4 \pi^{2} M_{1}} \sum_{q=1}^{\chi}\left(k_{\Delta}^{(q)} \otimes \Phi_{1,1}\right)} \\
\widetilde{F}=\frac{1}{M_{1}} \sum_{q=1}^{\chi}\left[\left(c_{x z}^{(q)} \otimes \phi_{1}\right) \dot{x}^{(q)}+\left(c_{z z}^{(q)} \otimes \phi_{1}\right) \dot{z}^{(q)}-\left(k_{\Delta}^{(q)} \otimes \phi_{1}\right) z^{(q)}\right]
\end{gathered}
$$

where $f_{1}$ and $\xi_{1}$ are fundamental frequency and damping of structure without including the crowd effect; $k_{\Delta}^{(q)} \otimes \Phi_{1,1}$ and $k_{\Delta}^{(q)} \otimes \phi$ are given by Equation (12).

$$
\begin{gathered}
k_{\Delta}^{(q)} \otimes \Phi_{1,1}=k_{\Delta l}^{(q)} \Phi_{1,1}\left(x_{l}^{(q)}\right)+k_{\Delta t}^{(q)} \Phi_{1,1}\left(x_{t}^{(q)}\right) \\
k_{\Delta}^{(q)} \otimes \phi_{1}=k_{\Delta l}^{(q)} \phi_{1}\left(x_{l}^{(q)}\right)+k_{\Delta t}^{(q)} \phi_{1}\left(x_{t}^{(q)}\right) \\
k_{\Delta l}^{(q)}=k_{l}^{(q)}\left(L_{0}^{(q)} / L_{l}^{(q)}-1\right), k_{\Delta t}^{(q)}=k_{t}^{(q)}\left(L_{0}^{(q)} / L_{t}^{(q)}-1\right)
\end{gathered}
$$


In general, the most unfavorable excitation induced by humans is that all pedestrians simultaneously walk with a uniform gait. It is very important for engineers to work out how to resist excessive vibration under this excitation. Calculating the dynamic properties induced by uniform excitation is necessary to control the excessive vibration of the structure. A further simplification is made in following section, so that the qualitative relationships between crowd size and modal properties can be estimated.

\section{Excitation Assumption}

All pedestrians are assumed to be uniformly distributed on a footbridge deck, without an overlap, as shown in Figure 2. Additionally, pedestrians are assumed to be moving with consistent walking behavior and that they have the same dynamic properties. For simplicity, the superscript " $(q)^{\prime \prime}$ is omitted in the following contents. Each pedestrian is assumed to walk in a fixed position, as shown in Figure 3. To be more specific, each pedestrian's walking behavior is similar to the movement on a treadmill. The numbers of pedestrians in longitudinal and lateral directions are $N_{x}$ and $N_{y}$, respectively. The total number of pedestrians can be obtained with $\chi=N_{x} N_{y}$.

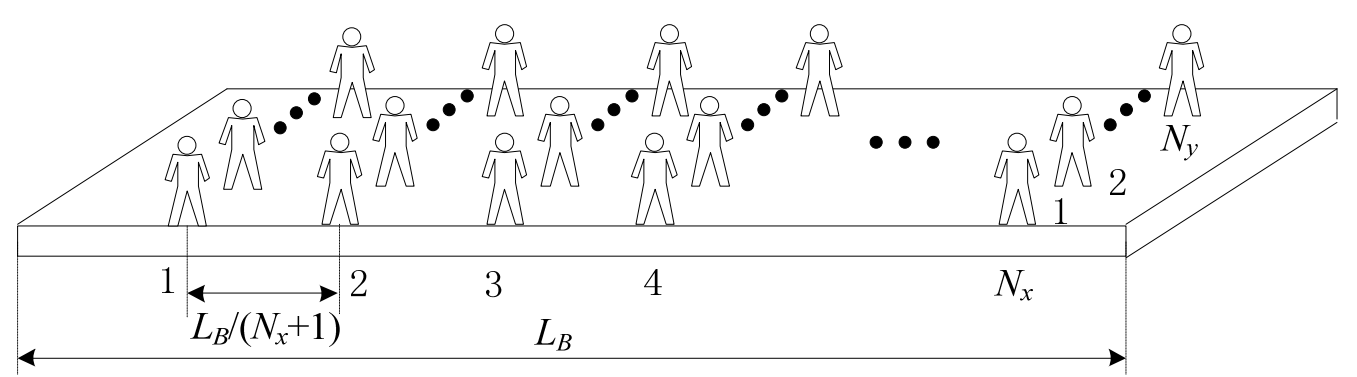

Figure 2. Diagram of a uniformly arranged crowd on a footbridge.

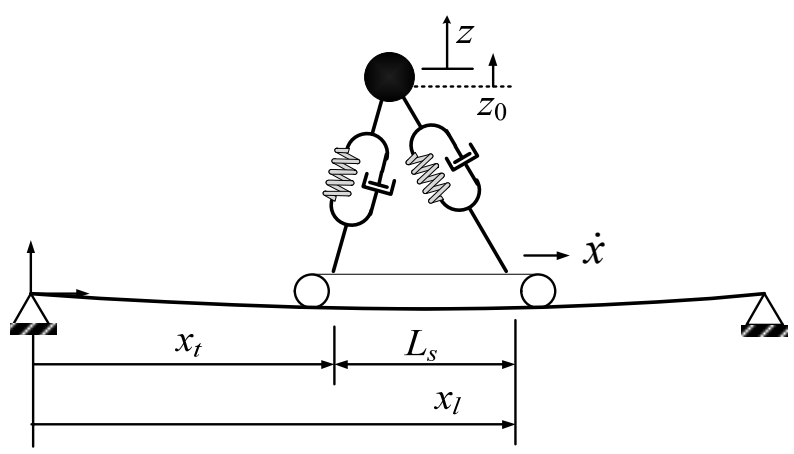

Figure 3. Assumption of stepping in a fixed position.

Considering the step length $L_{s}$ is much less than the structure span $L_{B}$, the models of two footholds are approximated as:

$$
\phi_{1}\left(x^{(q)}\right) \simeq \phi_{1}\left(x_{l}^{(q)}\right), \phi_{1}\left(x^{(q)}\right) \simeq \phi_{1}\left(x_{t}^{(q)}\right)
$$

Without considering leg compression, the lengths of two legs are kept as the original length. Based on the above assumption, the damping parameters $\sum_{q=1}^{\chi}\left(c_{z z}^{(q)} \otimes \Phi_{1,1}\right)$ can be approximated by:

$$
\sum_{q=1}^{\chi}\left(c_{z z}^{(q)} \otimes \Phi_{1,1}\right) \simeq N_{y} c_{l e g}\left(\frac{z}{L_{0}}\right) \sum_{q=1}^{N_{x}}\left(\sin ^{2}\left(\frac{q \pi}{N_{x}+1}\right)\right)
$$


where $c_{\text {leg }}$ is the damping of leg. The $\sum_{q=1}^{\chi}\left(k_{\Delta}^{(q)} \otimes \Phi_{1,1}\right)$ in Equation (10) is calculated by

$$
\begin{aligned}
\sum_{q=1}^{\chi}\left(k_{\Delta}^{(q)} \otimes \Phi_{1,1}\right) & \simeq N_{y} \sum_{q=1}^{N_{x}}\left(k_{\text {leg }}\left(\Delta L_{l} / L_{l}+\Delta L_{t} / L_{t}\right) \Phi_{1,1}\left(x^{(q)}\right)\right) \\
& \simeq 2 N_{y} k_{\operatorname{leg}} \frac{\Delta z}{L_{0}} \sum_{q=1}^{N_{x}} \sin ^{2}\left(\frac{q \pi}{N_{x}+1}\right)
\end{aligned}
$$

where $\Delta z$ is vertical vibration amplitude of CoM and $k_{\text {leg }}$ is leg stiffness.

$$
\sum_{q=1}^{\chi}\left(c_{x z}^{(q)} \otimes \phi_{1}\right) \dot{x}^{(q)}, \quad \sum_{q=1}^{\chi}\left(c_{z z}^{(q)} \otimes \phi_{1}\right) \dot{z}^{(q)} \quad \text { and } \quad \sum_{q=1}^{\chi}\left(k_{\Delta}^{(q)} \otimes \phi_{1}\right) z^{(q)} \quad \text { are } \quad \text { calculated by }
$$
Equations (18)-(20), respectively.

$$
\begin{aligned}
\sum_{q=1}^{\chi}\left(c_{x z}^{(q)} \otimes \phi_{1}\right) \dot{x}^{(q)} & \simeq \sum_{q=1}^{\chi}\left(c_{l x z}^{(q)}+c_{t x z}^{(q)}\right) \phi_{1}\left(x^{(q)}\right) \dot{x}^{(q)} \simeq N_{y} \frac{c_{l e g} z \dot{x}}{L_{0}^{2}}\left(2 x-x_{l}-x_{t}\right) \sum_{q=1}^{N_{x}} \sin \left(\frac{q \pi}{N_{x}+1}\right) \\
\sum_{q=1}^{\chi}\left(c_{z z}^{(q)} \otimes \phi_{1}\right) \dot{z}^{(q)} & \simeq N_{y} c_{l e g} \frac{z \dot{z}}{L_{0}} \sum_{q=1}^{N_{x}} \sin \left(\frac{q \pi}{N_{x}+1}\right) \\
\sum_{q=1}^{\chi}\left(k_{\Delta}^{(q)} \otimes \phi_{1}\right) z^{(q)} & \simeq N_{y} k_{l e g} \Delta z\left(\frac{1}{\sin \theta_{l}}+\frac{1}{\sin \theta_{t}}\right) \sum_{q=1}^{N_{x}} \sin \left(\frac{q \pi}{N_{x}+1}\right) \\
& \simeq N_{y} k_{l e g} \Delta z \sum_{q=1}^{N_{x}} \sin \left(\frac{q \pi}{N_{x}+1}\right)
\end{aligned}
$$

The $\sum_{q=1}^{\chi} \sin ^{2}\left(\frac{q \pi}{N_{x}+1}\right)$ is simplified by employing the Taylor expansion as:

$$
\begin{aligned}
& \sum_{q=1}^{N_{x}} \sin ^{2}\left(\frac{q \pi}{N_{x}+1}\right) \simeq\left(\frac{\pi}{N_{x}+1}\right)^{2} \sum_{q=1}^{N_{x}} q^{2}-\frac{1}{3}\left(\frac{\pi}{N_{x}+1}\right) \sum_{q=1}^{4} q^{4}+\frac{1}{36}\left(\frac{\pi}{N_{x}+1}\right) \sum_{q=1}^{6} q^{6} \\
& =\frac{\pi_{x}}{6} \frac{N_{x}\left(2 N_{x}+1\right)}{N_{x}+1}-\frac{\pi^{4}}{90} \frac{N_{x}\left(2 N_{x}+1\right)\left(3 N_{x}^{2}+3 N_{x}-1\right)}{\left(N_{x}+1\right)^{3}}+\frac{\pi^{6}}{1512} \frac{N_{x}\left(2 N_{x}+1\right)\left(3 N_{x}^{4}+6 N_{x}^{3}-3 N_{x}+1\right)}{\left(N_{x}+1\right)^{5}} \\
& \simeq\left(\frac{1}{3}-\frac{\pi^{2}}{15}+\frac{\pi^{4}}{252}\right) \pi^{2} N_{x}
\end{aligned}
$$

Similarly, the $\sum_{q=1}^{N_{x}} \sin \left(\frac{q \pi}{N_{x}+1}\right)$ is approximated as:

$$
\sum_{q=1}^{N_{x}} \sin \left(\frac{q \pi}{N_{x}+1}\right) \simeq \frac{\pi}{2}\left(1-\frac{\pi^{2}}{12}\right) N_{x}
$$

Substituting Equations (16) and (21) into Equation (9), the system damping ratio induced by the crowd can be estimated as:

$$
\widetilde{\xi} \simeq \xi_{1} \frac{f_{1}}{\widetilde{f}}+\left(\frac{1}{3}-\frac{\pi^{2}}{15}+\frac{\pi^{4}}{252}\right) \frac{\chi \pi c_{\text {leg }}}{4 M_{1} \widetilde{f}} \frac{z}{L_{0}}
$$

Similarly, by substituting Equations (17) and (21) into Equation (10), the system frequency induced by crowd is:

$$
\widetilde{f}=\sqrt{f_{1}^{2}-\left(\frac{1}{3}-\frac{\pi^{2}}{15}+\frac{\pi^{4}}{252}\right) \frac{\chi k_{l e g} \Delta z}{2 M_{1} L_{0}}}
$$


By substituting Equations (18)-(20) and (22) into Equation (11), the system excitation force induced by the crowd is calculated by:

$$
\widetilde{F}=\left(1-\frac{\pi^{2}}{12}\right) \frac{\pi \chi}{2 M_{1}}\left[\frac{c_{l e g} \dot{x} z}{L_{0}^{2}}\left(2 x-x_{l}-x_{t}\right)+\frac{c_{l e g} \dot{z} z}{L_{0}}-k_{l e g} \Delta z\right]
$$

Longitudinal movement velocity of the CoM is approximated to follow cosine [14]. The velocity of the CoM is at its highest when the CoM is approximately located at the mid-position between two footholds. In this study, longitudinal velocity of the CoM is defined as:

$$
\dot{x}(t)=\frac{L_{S}}{T_{S}}-A_{v x} \cos \left(\frac{2 \pi t}{T_{S}}\right),\left(0 \leq t<T_{S}\right)
$$

where $T_{S}$ is the period of stepping; $A_{v x}$ is the varying amplitude of the CoM velocity around mean velocity. Longitudinal displacement of the CoM can be obtained by Equation (27) after integration with Equation (26)

$$
x(t)=\frac{L_{s}}{T_{S}} t-\frac{A_{v} T_{s}}{2 \pi} \sin \frac{2 \pi t}{T_{S}}+\frac{x_{l}+x_{t}}{2}-\frac{L_{s}}{2},\left(0 \leq t<T_{S}\right)
$$

When the supporting leg is upright in the single stance phase, the CoM reaches the summit in the vertical direction. When the CoM is located at the mid-position between two footholds, it reaches the lowest place in the vertical direction [14]. The vertical movement trajectory of the CoM is approximately sinusoidal and its expression is defined as:

$$
z(t)=L_{0}-\Delta z_{g}-A_{z} \sin \frac{\pi t}{T_{s}},\left(0 \leq t<T_{s}\right)
$$

where $\Delta z_{g}$ is the compression deformation of the leg, induced by gravitational effect; $A_{z}$ is the vertical change amplitude of the CoM. The vertical vibration amplitude of the CoM $\Delta z$ can be obtained by:

$$
\Delta z(t)=L_{0}-z(t)=\Delta z_{g}+A_{z} \sin \frac{\pi t}{T_{s}},\left(0 \leq t<T_{s}\right)
$$

Vertical velocity of the CoM can be obtained after a derivative operation to Equation (30) as:

$$
\dot{z}(t)=-\frac{\pi A_{z}}{T_{s}} \cos \frac{\pi t}{T_{s}},\left(0 \leq t<T_{s}\right)
$$

Substituting Equations (26)-(30) into Equations (23)-(25), the damping ratio, frequency and excitation of the structure induced by the crowd can be expressed as:

$$
\begin{gathered}
\widetilde{\xi} \simeq \xi_{1} \frac{f_{1}}{\widetilde{f}}+\left(\frac{1}{3}-\frac{\pi^{2}}{15}+\frac{\pi^{4}}{252}\right) \frac{\chi \pi c_{l e g}}{4 M_{1} \widetilde{f}}\left(1-\frac{\Delta z_{g}+A_{z} \sin \pi f_{s} t}{L_{0}}\right) \\
\widetilde{f}=\sqrt{f_{1}^{2}-\left(\frac{1}{3}-\frac{\pi^{2}}{15}+\frac{\pi^{4}}{252}\right) \frac{\chi k_{l e g}}{2 M_{1}}\left(\frac{\Delta z_{g}+A_{z} \sin \pi f_{s} t}{L_{0}}\right)} \\
\widetilde{F}=\left(1-\frac{\pi^{2}}{12}\right) \frac{\pi \chi}{2 M_{1}}\left[\begin{array}{l}
\frac{c_{l e g}}{L_{0}^{2}}\left(L_{s} f_{s}-A_{v x} \cos 2 \pi f_{s} t\right)\left(L_{0}-\Delta z_{g}-A_{z} \sin \pi f_{s} t\right)\left(2 L_{s} f_{s} t-\frac{A_{v x} T_{s}}{\pi} \sin 2 \pi f_{s} t-L_{s}\right) \\
-\frac{\pi f_{s} A_{z} c_{l e g} \cos \left(\pi f_{s} t\right)\left(L_{0}-\Delta z_{g}-A_{z} \sin \pi f_{s} t\right)}{L_{0}}-k_{l e g}\left(\Delta z_{g}+A_{z} \sin \pi f_{s} t\right)
\end{array}\right]
\end{gathered}
$$

where $f_{s}=1 / T_{s}$ is step frequency.

The damping ratio of Equation (31) shows that crowd size has a positive effect on structural damping. The larger crowd size $\chi$ induces the larger damping of the structure. However, the effect of crowd size on structural frequency is the opposite, as shown in Equation (32). Increase of crowd size $\chi$ 
causes a decrease of structural frequency. In addition, structural modal characteristics are also related to the movement of pedestrians. The term $A_{z} \sin \pi f_{s} t$ can disturb the effect of crowds on structural dynamic properties.

\section{Numerical Validation}

A simply supported beam [15] with a span of $40 \mathrm{~m}$ is used for the simulation. Its mass per meter is $\bar{m}=2400 \mathrm{~kg} / \mathrm{m}$. Vertical flexural stiffness is $E I=9.96 \times 10^{9} \mathrm{Nm}^{2}$. Damping ratio is $\xi=1.0 \%$. Fundamental frequency of the beam is $2.0 \mathrm{~Hz}$. Parametric values of pedestrians are listed in Table 1.

Table 1. Parameters of the crowd.

\begin{tabular}{ccc}
\hline Parameters & Values & References \\
\hline$m$ & $80 \mathrm{~kg}$ & \\
$L_{0}$ & $1.0 \mathrm{~m}$ & \\
$k_{\text {leg }}$ & $20 \mathrm{kN} / \mathrm{m}$ & {$[4]$} \\
$\xi_{\text {leg }}$ & $8 \%$ & \\
$L_{s}$ & $0.7 \mathrm{~m}$ & {$[16]$} \\
$A_{v x}$ & $0.005 \mathrm{~m} / \mathrm{s}$ & {$[14]$} \\
$A_{z}$ & $0.002 \mathrm{~m}$ & \\
\hline
\end{tabular}

To contrast the effect of crowd size and step frequency, the dynamic properties under different crowd sizes and step frequencies are analyzed. When the mass ratio $M_{p} / M_{s}$ between crowd and structure increases from 0.1 to 0.5 under same step $1 \mathrm{~Hz}$ in Figure 4, the frequency approximately decreases from $1.985 \mathrm{~Hz}$ to $1.92 \mathrm{~Hz}$ and the damping ratio also shows an obvious change in Figure $4 \mathrm{~b}$. However, the change of step frequency under the same mass ratio $M_{p} / M_{s}=0.5$ has a smaller effect on the model parameters. Figure $4 c$ gives the detail of damping ratio under the $M_{p} / M_{s}=0.1$ and $f_{s}=1 \mathrm{~Hz}$, which shows that the structure damping ratio is periodically changed along with walking gait. The frequency in Figure 4a also shows this effect and model property is disturbed by periodic walking gaits. The crowd size has a more significant impact on model properties than the walking excitation frequency.

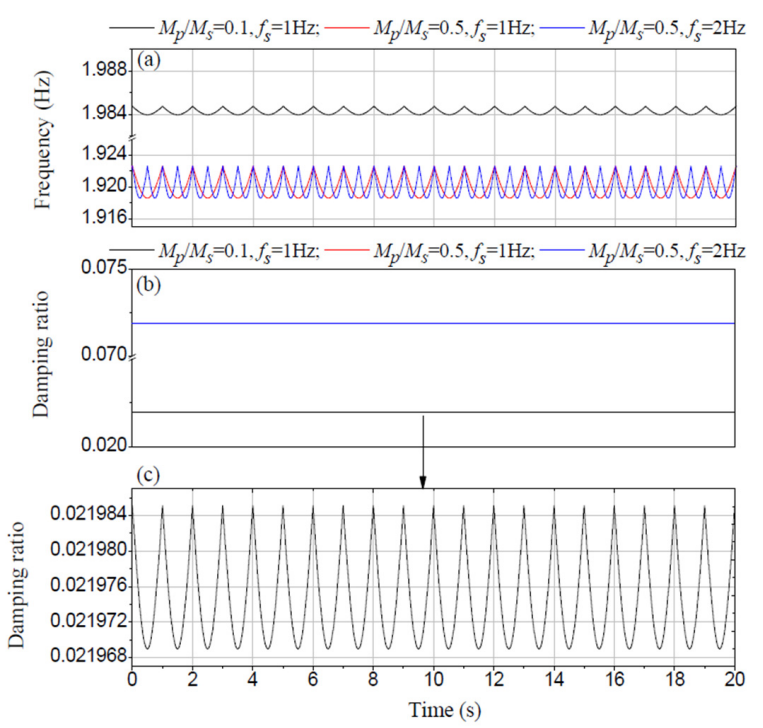

Figure 4. Effect of step frequency and crowd size on dynamic properties: (a) the larger mass ratio $M_{p} / M_{s}$ results the lower natural frequencies of structure, which is altered following the step rate; (b) the larger mass $M_{p} / M_{s}$ results the larger damping of structure; (c) damping of occupied structure is altered following the step rate $1 \mathrm{~Hz}$ under the mass ratio $M_{p} / M_{s}=0.1$. 
The dynamic responses in mid-span are given in Figure 5. Under the same walking frequency $f_{s}=1.0 \mathrm{~Hz}$, the change of mass ratio between crowd and structure has a tiny effect on vibration amplitude. However, the variation of step frequency has an obvious impact on structural responses. Under the walking frequency $f_{s}=2.0 \mathrm{~Hz}$, which is the same as the structural natural frequency, the acceleration response is rapidly amplified, as shown in Figure $5 \mathrm{~b}$. This demonstrates that significantly varying the frequency of excitation from the structural natural frequency is a more efficient method for alleviating excessive vibration, than by limiting the crowd size.

In order to explore the effect of crowd size, crowd mass was linearly increased from zero to half of the structure mass over $60 \mathrm{~s}$. Figure 6 gives the frequency and damping ratio under the resonance excitation frequency $f_{s}=2.0 \mathrm{~Hz}$. Along with the increase of mass ratio between crowd and structure, crowd induced frequency approximately decreases from $2 \mathrm{~Hz}$ to $1.92 \mathrm{~Hz}$ and damping ratio approximately increases from $1 \%$ to $7 \%$. It is noted that the both dynamic response displacement and acceleration amplitudes (Figure 7) increase along with the increase of mass ratio between crowd and structure under resonance excitation frequency. Thus it can be seen that the increase of crowd size under resonance excitation has the most prominent effect on structural dynamic properties.
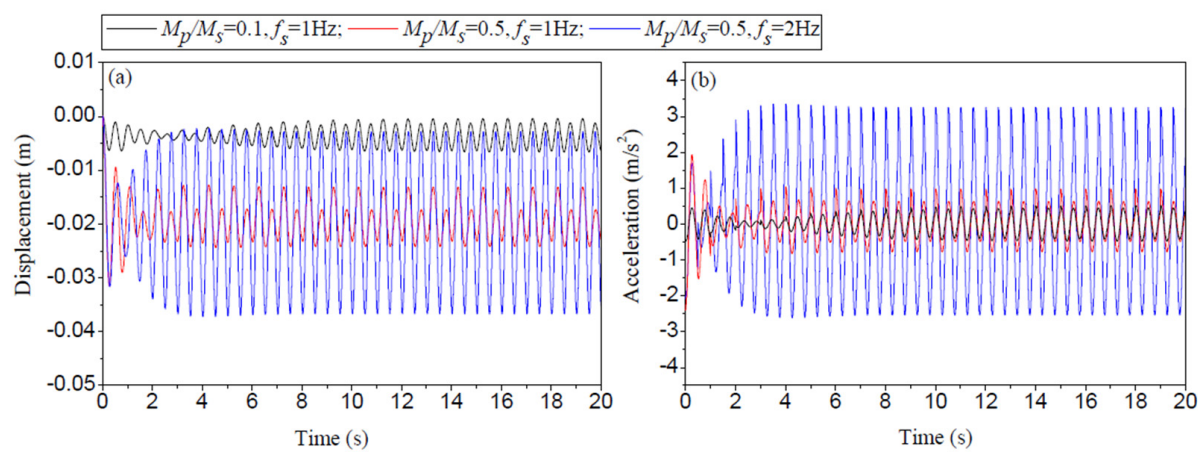

Figure 5. Effect of step frequency and crowd size on dynamic responses: (a) the larger mass ratio $\mathrm{Mp} / \mathrm{Ms}=0.5$ induces the slightly larger vibrational displacement under the uniform non-resonant excitation rate $f_{S}=1 \mathrm{~Hz}$; however the resonant excitation rate $f_{s}=2 \mathrm{~Hz}$ induces the noteworthy larger vibrational displacement amplitude under the same mass ratio $M_{p} / M_{s}=0.5$; (b) the larger mass ratio $M_{p} / M_{s}=0.5$ induces the slightly larger vibrational acceleration under the uniform non-resonant excitation rate $f_{s}=1 \mathrm{~Hz}$; but the resonant excitation rate $f_{s}=2 \mathrm{~Hz}$ induces the remarkable larger vibrational acceleration amplitude under the same mass ratio $M_{p} / M_{S}=0.5$.
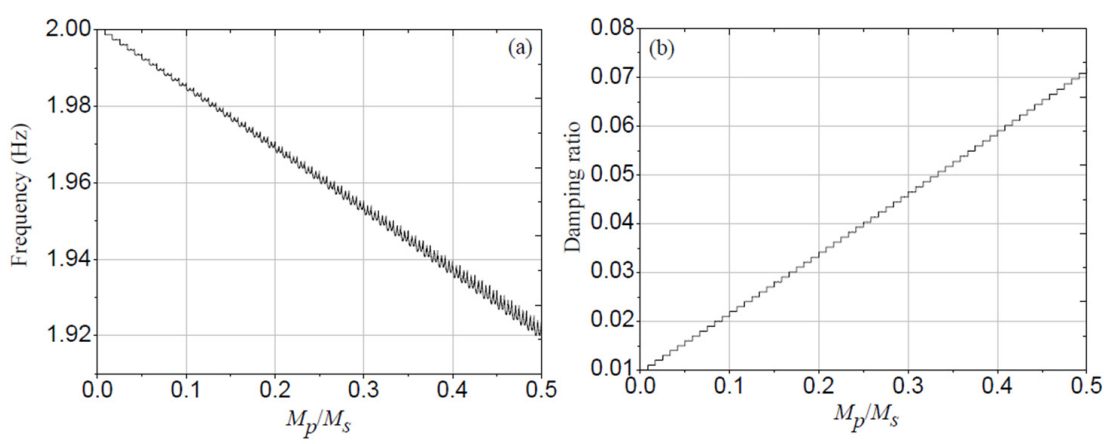

Figure 6. Effect of mass ratio between crowd and structure on dynamic properties: (a) the larger mass ratio between crowd and structure induces the lower frequency of structure under resonant excitation; (b) the larger mass ratio between crowd and structure results the larger damping of structure under resonant excitation. 

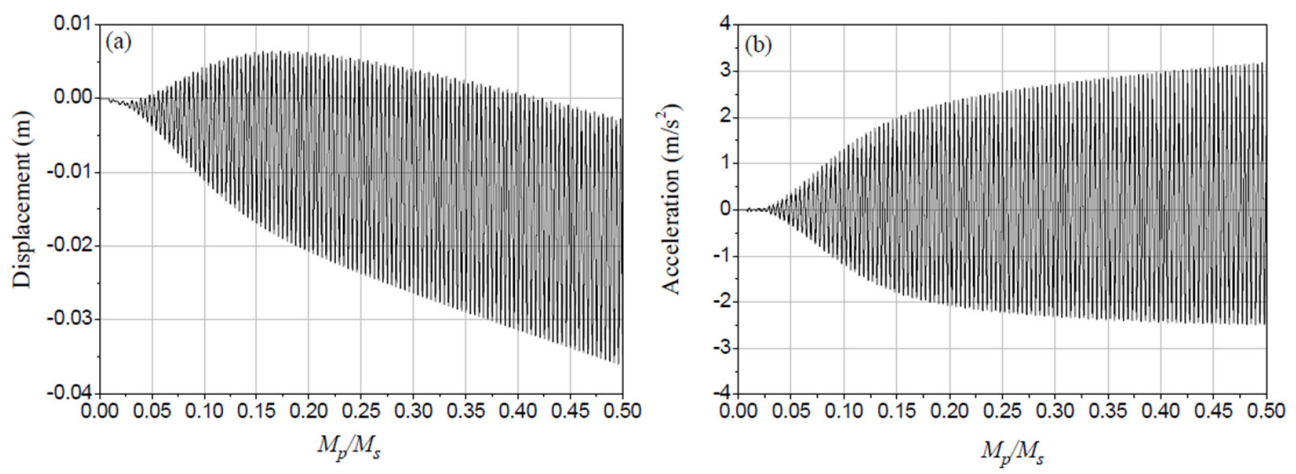

Figure 7. Effect of mass ratio between crowd and structure on dynamic responses: the increase of mass ratio induces corresponding persistent growth of structural vibrational displacement (a) and acceleration (b) under resonant excitation.

To further study the effect of crowd size, dynamic property peaks, including the frequency minimum peak, damping ratio maximum peak, and acceleration maximum peak were plotted, as shown in Figure 8. Along with the increase of mass ratio between crowd and structure, the frequency peak approximately decreases linearly, as shown in Figure 8a, which also happens to the damping ratio peak, as shown in Figure 8b. In Figure 8c, the acceleration peak shows a faster increase when the mass ratio $M_{p} / M_{s}$ is less than 0.3 . When the $M_{p} / M_{s}$ is larger than 0.3 , the acceleration approximately increases along with the increase of $M_{p} / M_{s}$. The influence of crowd size on model characteristics is consistent with measurement results [3].
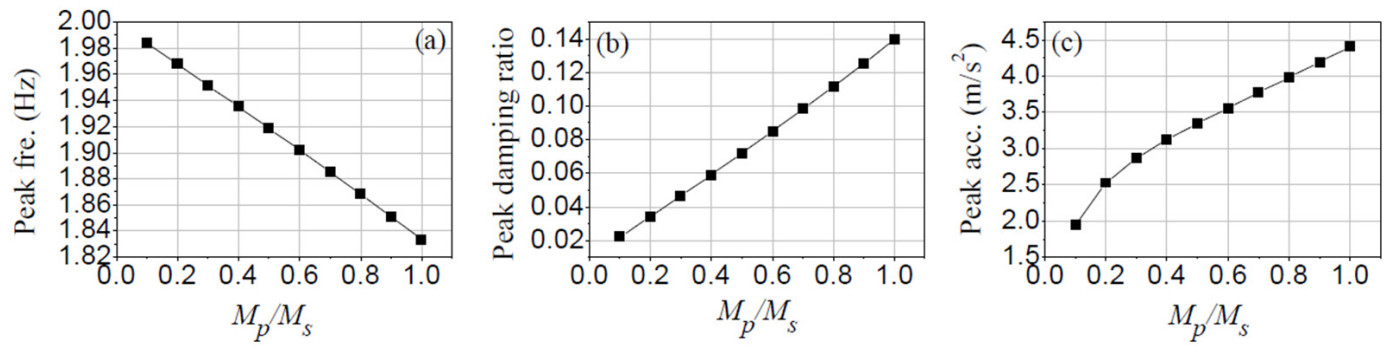

Figure 8. Dynamic property peaks under different mass ratios between crowd and structure: (a) structural frequency is approximately linearly decreased along with the growth of mass ratio; (b) structural damping is approximately linearly increased along with the growth of mass ratio; (c) the increase of mass ratio result in the persistent growth of peak acceleration under resonant excitation.

The model property formulas induced by crowd are obtained under the assumption that all pedestrians move consistently and that they follow a uniform distribution on the structure, which is also the most unfavorable situation for the control of structural vibration. However, the above descriptions do not happen frequently in real walking behaviors. Additionally, interactions between pedestrians may alleviate the worst situation. The proposed model formulas caused by crowds may give a more significant indication of human behaviors. Thus, a reduction coefficient needs to be incorporated into the model formulas and its definition needs experimentation, with the dynamic measurement of structure under the walking crowd in further research.

\section{Conclusions}

This paper proposes some formulas for vertical model property estimation, considering crowd size variation, based on a uniform distribution assumption with consistent walking behavior. The frequency and damping ratio of the structure can be obtained with a given crowd size. The frequency 
of the structure decreases, while the damping ratio of the structure increases along with the increase of crowd size. The influence of crowd actions is consistent with the dynamic measurement of the structure. The proposed model formulas induced by humans opens the field for further exploration on how walking crowds influence the vibration performances of these large-span structures.

Acknowledgments: This research is supported by the National Natural Science Foundation of China on Grant 51338001, the National Key Technology R\&D Program 2015BAK01B02 and Beijing Natural Science Foundation of China 8151003.

Author Contributions: Yan-an Gao conceived the idea and wrote the paper; Juan Wang and Min Liu analyzed the data and polished the writing.

Conflicts of Interest: The authors declare no conflict of interest.

\section{References}

1. Ellis, B.R.; Ji, T. Human-structure interaction in vertical vibration. Structure 1997, 122, 1-9. [CrossRef]

2. Busca, G.; Cappellini, A.; Manzoni, S.; Tarabini, M.; Vanali, M. Quantification of changes in modal parameters due presence of passive people on a slender structure. J. Sound Vib. 2014, 333, 5641-5652. [CrossRef]

3. Shahabpoor, E.; Pavic, A.; Racic, V.; Zivanovic, S. Effect of group walking traffic on dynamic properties of pedestrian structures. J. Sound Vib. 2017, 287, 207-225. [CrossRef]

4. Qin, J.W.; Law, S.S.; Yang, Q.S.; Yang, N. Pedestrian-bridge dynamic interaction, including human participation. J. Sound Vib. 2013, 332, 1107-1124. [CrossRef]

5. Qin, J.W.; Law, S.S.; Yang, Q.S. Finite element analysis of pedestrian-bridge dynamic interaction. J. Appl. Mech. 2014, 81, 041001. [CrossRef]

6. Zhou, D.; Ji, T. Dynamic characteristics of a beam and distributed spring-mass system. Int. J. Solids Struct. 2006, 43, 5555-5569. [CrossRef]

7. Zhou, D.; Han, H.; Ji, T.; Xu, X. Comparison of two models for human-structure interaction. Appl. Math. Model. 2016, 40, 3738-3748. [CrossRef]

8. Caprani, C.C.; Ahmadi, E. Formulation of human-structure interaction system models for vertical vibration. J. Sound Vib. 2016, 377, 346-367. [CrossRef]

9. Nimmen, K.V.; Lombaert, G.; Roeck, G.D.; Broeck, P.V. Reduced-order models for vertical human-structure interaction. J. Phys. Conf. Ser. 2016, 744, 012030. [CrossRef]

10. Alonso, J.F.J.; Saez, A.; Caetano, E.; Magalhaes, F. Vertical crowd-structure interaction model to analyze the change of the modal properties of a footbridge. J. Bridge Eng. 2016, 21, C4015004. [CrossRef]

11. Gao, Y.; Yang, Q. A walking crowd-structure interaction model. J. Vib. Shock 2016, 35, 153-159.

12. Gao, Y.A.; Yang, Q.S.; Qin, J.W. Bipedal crowd-structure interaction including social force effects. Int. J. Struct. Stab. Dyn. 2017, 17. [CrossRef]

13. Gao, Y.A.; Yang, Q.S. A theoretical treatment of crowd-structure interaction. Int. J. Struct. Stab. Dyn. 2018, 28, 1871001. [CrossRef]

14. Liu, J.; Urbann, O. Bipedal walking with dynamic balance that involves three-dimensional upper bodymotion. Robot. Auton. Syst. 2016, 77, 39-54. [CrossRef]

15. Li, Q.; Fan, J.; Nie, J.; Li, Q.; Chen, Y. Crowd-induced random vibration of footbridge and vibration control using multiple tuned mass dampers. J. Sound Vib. 2010, 329, 4068-4092. [CrossRef]

16. Hof, A.L.; Vermerris, S.M.; Gjaltema, W.A. Balance responses to lateral perturbations in human treadmill walking. J. Exp. Biol. 2010, 213, 2655-2664. [CrossRef] [PubMed] 